\title{
A proposal for a new type of thin-film field-emission display by edge breakdown of MIS structure
}

\author{
V.N. Konopsky \\ Institute of Spectroscopy, Russian Academy of Sciences, \\ Troitsk, Moscow region, 142092, Russia. \\ (e-mail: konopsky@isan.troitsk.ru)
}

\begin{abstract}
A new type of field emission display (FED) based on an edge-enhance electron emission from metal-insulator-semiconductor (MIS) thin film structure is proposed. The electrons produced by an avalanche breakdown in the semiconductor near the edge of a top metal electrode are initially injected to the thin film of an insulator with a negative electron affinity (NEA), and then are injected into vacuum in proximity to the top electrode edge. The condition for the deep-depletition breakdown near the edge of the top metal electrode is analytically found in terms of ratio of the insulator thickness to the maximum (breakdown) width of the semiconductor depletition region: this ratio should be less than $2 /(3 \pi-2) \simeq 0.27$. The influence of a neighboring metal electrode and an electrode thickness on this condition are analyzed. Different practical schemes of the proposed display with a special reference to $\mathrm{M} / \mathrm{CaF}_{2} / \mathrm{Si}$ structure are considered.
\end{abstract}

\section{Introduction}

Flat panel field emission displays have the potential to be a low cost, high performance alternative to the currently dominant cathode ray tube and liquid crystal displays. The first major problem in FEDs is the development of a reliable and efficient cold cathode electron emitter. Current FED prototypes use sharp metal or semiconductor tips as field emitters [1], that requires expensive lithography and other difficult fabrication processes. Besides, the 
control voltage for such tip emitters is rather high (about $100 \mathrm{~V}$ ). Some researchers use diamond-like films that contain nanoscale crystalline structure as the electron source [2], or use diamond and other coatings of the sharp tips to improve the emission properties of the tips [3, 团.

In the present paper we propose another type of field emitter, where electrons are produced by an avalanche breakdown in the semiconductor near the edge of the top metal electrode in the MIS structure.

The plan of this paper is as follows: in Section 2 we describe the proposed display, based on the edge breakdown of the MIS structure. In Section 3 we discuss the condition under which edge breakdown in the MIS structure takes place. In Section 4 we estimate the influence of a neighboring electrode on the edge breakdown condition and in Section 5 we discuss and summarize our results. In Appendix we briefly estimate the influence of an electrode thickness on the edge breakdown condition.

\section{Field emission display based on edge breakdown of MIS structure}

The display proposed is schematically shown in Figure 1(a). Glass substrate 1 with conductive metal column lines 2 is coated with a thin semiconductor layer 3 , which contains low p-doped column lines 4 that coincide with the metal ones. The film of the insulator with NEA 5 is grown on semiconductor, and conductive metal row lines 6 are deposited on the insulator film. Above the MIS structure the fluorescent screen 7 is located. The anode (fluorescent screen) and cathode (MIS structure) regions are separated by a vacuum space 8 .

When a positive voltage pulse of duration which is short compared to the time constant of thermal generation of minority carriers (electrons in our case) is applied to the top electrode of the MIS structure, no inversion layer can form. Thus, a large potential drop across the semiconductor will take place. If the amplitude of the pulse voltage is increased, band bending reaches large values where minority carriers are generated by nonthermal effects (by avalanche in our case). The amplitude of the pulse voltage, at which avalanche occurs we designate by $V_{\text {break. }}$.

When we apply a pulse voltage $V_{\text {control }}$, which amplitude is less then $V_{\text {break }}$, but more than $V_{\text {break }} / 2$ to one row line, and simultaneously apply such a pulse voltage with another polarity 
to one column line, the avalanche breakdown in the semiconductor will take place at the intersection of these lines. Under condition, which will be pointed out below, the breakdown will take place near the edges of metal lines. Inasmuch as velocities of the avalanche electrons at the semiconductor-insulator interface are directed in an arbitrary way, it is clear that at least a portion of the avalanche electrons, ballistically passing through the thin film of the insulator, will be extracted with a good efficiency (due to NEA of the insulator) into vacuum and will not impinge on the top metal layer. Then this portion of electrons will be accelerated by screen voltage $V_{\text {screen }}(\sim+100 \div+1000 \mathrm{~V})$ and will be hit to the fluorescent screen as it is shown in Figure 1(b).

\section{Edge breakdown condition}

The deep-depletition breakdown of MOS capacitors was first reported by Goetsberger and Nicollian [5, 6], who experimentally investigated doping conditions under which uniform avalanche takes place. Later a "universal and normalized" criterion for "field uniformity" in MOS capacitors was offered in the form $d / W_{\max }>0.3$ (where $d$ is the insulator thickness and $W_{\max }$ is the maximum (breakdown) width of the semiconductor depletition region) [7, 8]. This criterion was suggested in [7] based on computer-calculated values of the field distribution in MOS capacitors.

In this section we obtain this criterion in an analytical form, with an emphasis on physical explanation of the result, that helps us to analyze operating conditions and an ultimate resolution of the display proposed.

For this purpose let us at first consider a simple two-dimensional model of the electric field near the edge of the metal plate (see Figure 2(a)). Here $A_{1}-A_{2}$ and $A_{2}-A_{3}-A_{4}$ are conductive plates. Let one plate have the potential $V$, and the other plate have the zero potential.

We will use a method of conformal transformations (see [9 or any textbook in this field) to find an electrical field distribution in such a system. The Schwarz-Chrisoffel transform

$$
z=\frac{1 h}{\pi}\left[2 \sqrt{\omega-1}-1 \ln \left(\frac{1-1 \sqrt{\omega-1}}{1+1 \sqrt{\omega-1}}\right)\right]
$$

relates the upper half $\omega$ plane (Figure 2(b)) to the interior of the region $A_{1}-A_{2}-A_{3}-A_{4}$ of the $z$ plane (Figure 2(a)) [10]. Thus, half of the real axis $\Re(\omega)>0$ is at potential $V$ while 
$\Re(\omega)<0$ is at zero potential. The potential of the electric field in the $\omega$ plane is the real part of the complex potential given by the analytical function

$$
F=\varphi+1 \psi=\frac{V}{1 \pi} \ln \omega .
$$

The electric field in the $z$ plane is

$$
\frac{d F}{d z}=\frac{\partial \varphi}{\partial x}-1 \frac{\partial \varphi}{\partial y}=E_{x}-{ }_{1} E_{y}=\frac{d F}{d \omega} \frac{1}{d z / d \omega} .
$$

Performing the differentiation with respect to $\omega$ in (11) and (2)

$$
\begin{gathered}
\frac{d z}{d \omega}=\frac{1 h}{\pi} \frac{\sqrt{\omega-1}}{\omega} \\
\frac{d F}{d \omega}=\frac{V}{1 \pi \omega}
\end{gathered}
$$

we get

$$
E_{x}-{ }_{1} E_{y}=-\frac{V}{h \sqrt{\omega-1}} .
$$

Our main interest is the field near $z=0(\omega=1)$. Expanding logarithm in (11) in a Taylor series at $\omega=1$ and holding the first two terms, we obtain

$$
\sqrt{\omega-1}=\left(\frac{3 \pi}{2 h 1} z\right)^{1 / 3}
$$

and from (6) we have

$$
E_{x}-{ }_{1} E_{y}=-\frac{V}{h\left(3 \pi z / 2 h_{1}\right)^{1 / 3}}
$$

or

$$
|E|=\frac{V}{h} \frac{(2 h / 3 \pi)^{1 / 3}}{\left(x^{2}+y^{2}\right)^{1 / 6}} .
$$

We may define

$$
z_{\mathrm{cr}}=\frac{2 h}{3 \pi} \simeq \frac{h}{4.71}
$$

as a "critical" distance from the edge at which field strength is still higher than $V / h$ - the field strength between the plates far from the edge.

Now the condition for edge breakdown is rather obvious: if insulator thickness is less than $z_{\text {cr }}$ (as is shown in Figure 3) the breakdown takes place near the edge. But if $d>z_{\text {cr }}$ the uniform breakdown occurs. The value $d+W_{\max }$ in the real MIS structure plays the role of the distance $h$ between the conductive plates in our model consideration. (The exception is the 
case when the distance $L$ (see Figure $1(\mathrm{~b})$ ) between the real metal plates is less than $d+W_{\max }$. In this case $h=L$.) From the equations

$$
\begin{aligned}
& h=d+W_{\max } \\
& d<z_{\text {cr }}=\frac{2 h}{3 \pi}
\end{aligned}
$$

we may obtain the criteria for the deep-depletition edge breakdown in terms of ratio of the insulator thickness to the maximum (breakdown) width of the semiconductor depletition region:

$$
\frac{d}{W_{\max }}<\frac{2}{3 \pi-2} \simeq 0.27
$$

One can see that the value obtained $(0.27)$ is consistent with the value 0.3 obtained in the work [7] by numerical computer-aided calculations for particular system $\mathrm{M} / \mathrm{SiO}_{2} / \mathrm{Si}$.

The model considered is applicable to real systems subject to the condition that the radius of the edge curvature of the top electrode is less than insulator thickness.

\section{Breakdown condition for double-edge structure}

In this Section we apply analogous approach to find breakdown condition for the system illustrated in Figure 4(a). It is necessary for several reasons: first, one may like to initiate electron emission into a slit in the top electrode. Second, it may be convenient to apply a voltage simultaneously to a set of neighboring row and column lines for more stable emission. And third, minimal distance between top electrodes defines the ultimate resolution of the proposed display.

The transformation

$$
z=\frac{21}{\pi}\left[s \operatorname{arctanh}\left(\frac{\omega}{\sqrt{\omega^{2}-\lambda^{2}}}\right)+h \arctan \left(\frac{h}{s} \frac{\omega}{\sqrt{\omega^{2}-\lambda^{2}}}\right)\right]
$$

where

$$
\lambda=\sqrt{1+\frac{h^{2}}{s^{2}}}
$$

relates the upper half $\omega$ plane (Figure $4(\mathrm{~b})$ ) to the interior of the region $A_{1}-A_{2}-A_{3}-A_{4}-$ $A_{5}-A_{6}$ of the $z$ plane (Figure 4(a)) [11]. Thus, parts of the real axis $\Re(\omega)<-1$ and $\Re(\omega)>1$ are at potential $V$ while part $-1<\Re(\omega)<1$ is at zero potential. The potential of the electric field in the $\omega$ plane is the real part of the complex potential given by the analytical function

$$
F=\frac{V}{1 \pi} \ln (w-1)-\frac{V}{1 \pi} \ln (w+1) .
$$


Using the differentials with respect to $\omega$ of (14)

$$
\frac{d z}{d \omega}=\frac{21 s}{\pi} \frac{\sqrt{\omega^{2}-\lambda^{2}}}{\left(\omega^{2}-1\right)}
$$

and (16)

$$
\frac{d F}{d \omega}=\frac{2 V}{1 \pi\left(\omega^{2}-1\right)}
$$

we may find the electric field in the $z$ plane from (3).

$$
E_{x}-1 E_{y}=-\frac{V}{s \sqrt{\omega^{2}-\lambda^{2}}} .
$$

Our concern is the field near $z=1 h \pm s(\omega= \pm \lambda)$ points. Expanding arctanh and arctan in (14) in a power series at $\omega=\lambda$ and holding the first two terms, we have

$$
\sqrt{\omega^{2}-\lambda^{2}}=\frac{h}{s}\left[\frac{3 \pi}{2 h 1}(z+h-1 s)\left(1+\frac{h^{2}}{s^{2}}\right)^{2}\right]^{1 / 3} .
$$

and from (19) we have

$$
E_{x}-1 E_{y}=-\frac{V}{h\left[\left(3 \pi / 2 h_{1}\right)(z+h-1 s)\left(1+h^{2} / s^{2}\right)^{2}\right]^{1 / 3}} .
$$

Now the "critical" distance from the edge at which field strength is still higher than $V / h$ is

$$
z_{\mathrm{cr} 2}=\frac{2 h}{3 \pi\left(1+h^{2} / s^{2}\right)^{2}} .
$$

One can see that this value is coincident with (10) when $s \gg h$.

For example, if $s=h$ so $z_{\text {cr2 }}$ is

$$
z_{\mathrm{cr} 2}=\frac{z_{\mathrm{cr}}}{4}=\frac{h}{6 \pi} .
$$

One can see that the distance $2 s$ between the top electrodes should be more than twice larger than $h=d+W_{\max }$ to avoid drastic decrease of $z_{\text {cr2 }}$.

\section{Discussion and summary}

Firstly, let us mention the kind of insulator that may be used in this display. At least three kinds of insulators are appropriate for our purpose: hydrogen-terminated diamond (111), LiF 
and $\mathrm{CaF}_{2}$. Diamond has long attracted considerable attention as a cold cathode for FED due to its NEA and robust mechanical and chemical properties.

$\mathrm{LiF}$ has the largest NEA of any solid. The NEA of LiF crystal is $-2.7 \mathrm{eV}$ [12]. The possibility of epitaxial grown of LiF films on the Ge was also reported in [12].

But we assume that the $\mathrm{CaF}_{2} / \mathrm{Si}$ system is the best choice. The reasons for this are as follows:

1. $\mathrm{CaF}_{2}$ has a small but negative electron affinity [13.

2. A very attractive property of $\mathrm{CaF}_{2}$ is similarity of its crystal structure to $\mathrm{Si}$ : $\mathrm{CaF}_{2}$ has cubic $\mathrm{m} 3 \mathrm{~m}$ structure with only $0.6 \%$ of lattice mismatch with $\mathrm{Si}(111)$ at room temperature. This fact makes it possible to grow perfect single crystal films of $\mathrm{CaF}_{2}$ on silicon by molecular beam epitaxy technique [14, 15]. The crystalline quality, chemical stability and electrical characteristics of $\mathrm{CaF}_{2}$ films grown on $\mathrm{Si}(111)$ may be further improved by rapid thermal annealing [16].

3. Barrier height between the conductive band minimum of Si and the conductive band minimum of $\mathrm{CaF}_{2}$ is only $\sim 2.2 \mathrm{eV}[15]$.

4. An additional attractive property of $\mathrm{CaF}_{2}$ is anomalously large low-energy electron escape depth of the order of $260 \mathrm{~nm}$ [17].

5. And lastly, the good emission properties of the $\mathrm{CaF}_{2} / \mathrm{Si}$ structure has recently been demonstrated in the experiment 4 .

The thickness of the insulator film about $d \sim 10 \mathrm{~nm}$ seems to be nearly optimum because it is large enough to avoid direct tunneling of electrons from semiconductor to metal, but it is small enough for ballistic passing of electrons through insulator film.

So, if one has $10 \mathrm{~nm}$ thick $\mathrm{CaF}_{2}$ film on the $1 \mu \mathrm{m}$ thick Si layer with p-dopant concentration $n \sim 10^{16} \mathrm{~cm}^{-3}\left(W_{\max } \sim 1 \mu \mathrm{m}[\mathbb{8}]\right)$, one may be sure that when pulse voltages with the amplitude $V_{\text {control }} \sim \pm 10 \mathrm{~V}$ are applied to one row and one column lines an avalanche breakdown will occur at the edges of metal lines $\left(d / W_{\max } \sim 0.01\right)$. The control voltage may be still decreased if the distance between metal electrodes $L$ will be less than $d+W_{\max }$ (but $L$ must always be more than $3 \pi d / 2$ ). The avalanche electrons will be injected into insulator at the $\alpha-\beta$ line, which shown in Figure 3. From geometrical considerations, up to several tens percent of electrons may be injected into vacuum. 
To summarize, we have proposed a simple design of the field-emission display based on the deep-depletition edge avalanche breakdown of the MIS structure. The control voltage of this display may be as small as tens volts and its ultimate resolution may reach several microns.

\section{Appendix}

Here we mention the influence of the top electrode thickness on the edge breakdown condition. To avoid complicated expressions, we give only a simple estimation of the effect by solving the problem presented in Figure 5(a), where top electrode has zero thickness. The equation

$$
z=\frac{h}{\pi}(\omega-\ln (w)-1)
$$

relates the upper half $\omega$ plane (Figure $5(\mathrm{~b})$ ) to the interior of the region $A_{1}-A_{2}-A_{3}-A_{4}$ of the $z$ plane (Figure 5(a)). The potential of the electric field in the $\omega$ plane is the real part of the complex potential given by the analytical function

$$
F=\frac{V}{1 \pi} \ln \omega .
$$

The electric field in the $z$ plane is

$$
\frac{d F}{d z}=\frac{d F}{d \omega} \frac{1}{d z / d \omega} .
$$

Performing the differentiation with respect to $\omega$ in (24) and (25)

$$
\begin{gathered}
\frac{d z}{d \omega}=\frac{h}{\pi} \frac{\omega-1}{\omega} \\
\frac{d F}{d \omega}=\frac{V}{1 \pi \omega}
\end{gathered}
$$

we get

$$
E_{x}-{ }_{1} E_{y}=\frac{V}{1 h(\omega-1)} .
$$

Our main interest is the field near $z=0(\omega=1)$. Expanding logarithm in (24) in a Taylor series at $\omega=1$ and holding the first two terms, we obtain

$$
\omega-1=\left(-\frac{2 \pi}{h} z\right)^{1 / 2}
$$

and from (6) we have

$$
E_{x}-{ }_{1} E_{y}=-\frac{V}{h(2 \pi z / h)^{1 / 2}} .
$$


One can see that the "critical" distance in this case is:

$$
z_{\mathrm{cr} 0}=\frac{h}{2 \pi} .
$$

And the edge breakdown condition is

$$
\frac{d}{W_{\max }}<\frac{1}{2 \pi-1} \simeq 0.19 .
$$

This edge breakdown condition should be used when top electrode thickness is less than any other dimensions in the system. Essentially it means that top electrode thickness should be less than thickness of the insulator film.

\section{References}

[1] Brodie I and Spindt C A 1992 Adv. Electron. Electron Phys. 831

[2] Zhu W, Kochansky G P, Jin S, Seibles L, Jacobson D C, McCormack M and White A E 1995 Appl. Phys. Lett. 671157

[3] Liu J, Zhirnov V V, Choi W B, Wojak G J, Myers A F, Cuomo J J and Hren J J 1996 Appl. Phys. Lett. 694038

[4] Konopsky V N, Zhirnov V V, Sokolov N S, Alvarez J C, Givargizov E A, Bormatova L V, Letokhov V S and Sekatskii S K 1996 J. Physique IV 6 C5-129

[5] Goetzberger A and Nicollian E H 1966 Appl. Phys. Lett. 9444

[6] Goetzberger A and Nicollian E H 1966 J. Appl. Phys. 384582

[7] Rusu A and Bulucea C 1979 IEEE Trans. Electron Devices ED-26 201

[8] Sze S M 1981 Physics of Semiconductor Devices (New York: A Willey - Interscience Publication John Wiley and Sons)

[9] Panofsky W K H and Phillips M 1968 Classical Electricity and Magnetism (Cambridge: Addison - Wesley Publishing Company, Inc.)

[10] Korn G A and Korn T M 1968 Mathematical Handbook (McGraw - Hill Book Company) 
[11] Lavrent'ev M A and Shabat B V 1965 Metody Teorii Funktsii Kompleksnogo Peremennogo (Moscow: Nauka) p 189 (in Russian)

[12] Lapiano-Smith D A, Eklund E A and Hipsel F J 1991 Appl. Phys. Lett. 592174

[13] Poole R T, Williams D R, Riley J D, Jenkin J G, Liesegang J and Leckey R C G 1975 Chem. Phys. Lett. 36401

[14] Batstone J L, Philips J M and Hunke E C 1988 Phys. Rev. Lett. 601394

[15] Rieger D, Himpsel F L, Karlsson U O, McFeely F R, Morar J F and Yarmoff J A 1986 Phys. Rev. B $\mathbf{3 4} 7295$

[16] Phillips J M, Manger M L, Pfeiffer L, Joy D C, Smith III T P, Augustyniak W M and West K M 1986 Mat. Res. Soc. Symp. Proc. 53155

[17] Quiniou B, Schwarz W, Wu Z, Osgood R M, Yang Q and Philips J M 1992 Appl. Phys. Lett. 60183 


\section{Figures' Captions}

Figure 1: Sectional view (a) and side view (b) of the FED based on edge breakdown of MIS structure. 1 - glass substrate, 2 - metal column lines, 3 - semiconductor layer, 4 p-doped column lines, 5 - insulator with NEA, 6 - metal row lines, 7 - fluorescent screen, 8 - vacuum spacing.

Figure 2: Plane $z$ (a) and plane $\omega$ (b) related by the equation (1)

Figure 3: Distance $z_{\text {cr }}$ and electron emission area $\alpha-\beta$ near the edge.

Figure 4: Plane $z(\mathrm{a})$ and plane $\omega(\mathrm{b})$ related by the equation (14)

Figure 5: Plane $z$ (a) and plane $\omega$ (b) related by the equation (24) (Appendix) 
This figure "figure1.gif" is available in "gif" format from: http://arxiv.org/ps/physics/9901018v1 
This figure "figure2.gif" is available in "gif" format from: http://arxiv.org/ps/physics/9901018v1 
This figure "figure3.gif" is available in "gif" format from: http://arxiv.org/ps/physics/9901018v1 
This figure "figure4.gif" is available in "gif" format from: http://arxiv.org/ps/physics/9901018v1 
This figure "figure5.gif" is available in "gif" format from: http://arxiv.org/ps/physics/9901018v1 